\title{
Circo y teatro para la transformación social: Repertorios de conocimiento y acción en la construcción de entornos protectores y la promoción de derechos de los niños, niñas y adolescentes
}

\section{Artículo de investigación}

\section{(c) (i)}

https://creativecommons.org/licenses/by/4.0/deed.es

\section{Alejandro Balanzó}

Universidad Externado de Colombia

alebalanzo@gmail.com

\section{Angie Paola Ariza Porras}

Universidad Autónoma de Madrid

investigacion@laventanaproducciones.co

\section{David Quiroga}

Universidad Colegio Mayor de Cundinamarca

sistematizacion.circopolis@gmail.com

\section{Ángela Gómez Ramírez}

Universidad Santo Tomás

ps.angelagomezramirez@gmail.com

Recibido: junio 1 de 2019

Aprobado: julio 16 de 2019

Cómo citar este artículo: Balanzó, A., Ariza Porras, A., Quiroga, D., y Gómez, A. (2020). Circo y teatro para la transformación social: Repertorios de conocimiento y acción en la construcción de entornos protectores y la promoción de derechos de los niños, niñas y adolescentes. Calle 14: revista de investigación en el campo del arte 15(27). pp. 168-181. DOI: https://doi. org/10.14483/21450706.15419

Agradecimientos: Artículo de investigación realizado en el marco del proyecto Circópolis - Transformado espacios y creando sueños, desarrollado como parte de la Estrategia de Acciones Masivas de Alto Impacto Social (AMAS) del Instituto Colombiano de Bienestar Familiar (ICBF) en alianza con la Corporación Producciones La Ventana. 
Circo y teatro para la transformación social: Repertorios de conocimiento y acción en la construcción

de entornos protectores y la promoción de derechos de los niños, niñas y adolescentes

\title{
Resumen
}

¿Cómo pueden el circo y el teatro aportar a la transformación hacia una paz fundada en la promoción de los derechos? ¿Qué lecciones nos han dejado las prácticas circenses y teatrales en contextos que han sido históricamente afectados por el conflicto armado? ¿Qué necesitan el teatro, el circo y el clown para ser medios que faciliten la construcción de entornos protectores para la niñez y la adolescencia en estos territorios? Este artículo aborda estas preguntas desde la experiencia del programa Circópolis, transformado espacios y creando sueños, en territorios afectados por el conflicto armado. Presentamos una interpretación de la sistematización de experiencias del programa a la luz del enfoque posturas de conocimiento de Balanzó en aras de identificar repertorios que tienen lugar cuando el trabajo artístico se enmarca en procesos de trabajo institucional ligados a discursos de política pública.

\section{Palabras clave}

Circo; teatro; clown; construcción de paz; derechos humanos; prevención del consumo de sustancias psicoactivas; posturas de conocimiento

Circus and Theater for Social Transformation: Repertoires of Knowledge and Action in the

Construction of Protective Environments and the Promotion of the Rights of Children and Adolescents

\begin{abstract}
How can circus and the theater contribute to a social transformation towards peace founded on the promotion of rights? What lessons have we received from circus and theater practices in contexts that have historically been affected by the armed conflict? What do theater, circus and clown need in order to become means that facilitate the construction of protective environments for children and adolescents in these territories? This article addresses these questions from the experience of the program Circópolis, transformando espacios y creando sueños, implemented in territories affected by the armed conflict. We present an interpretation of the systematization of program experiences in the light of Balanzó's knowledge stances perspective, in order to identify repertoires that take place when an artistic work is framed in institutional work processes linked to public policy discourses.
\end{abstract}

\section{Keywords}

Circus; theater; clown; peace building; human rights; prevention of the consumption of psychoactive substances; knowledge stances

\section{Cirque et théâtre pour la transformation sociale : Répertoires de connaissances et d'action dans la construction d'environnements protecteurs et dans la promotion des droits des enfants et des}

\section{Résumé}

Comment le cirque et le théâtre peuvent-ils contribuer à une transformation sociale vers une paix fondée sur la promotion des droits ? Quelles leçons avons-nous tirées des pratiques de cirque et de théâtre dans des contextes historiquement affectés par le conflit armé ? De quoi le théâtre, le cirque et le clown ont-ils besoin pour devenir des moyens facilitant la construction d'environnements protecteurs pour les enfants et les adolescents de ces territoires ? Cet article aborde ces questions à partir de l'expérience du programme Circópolis, transformando espacios y creando sueños, mis en œuvre dans des territoires touchés par le conflit armé. Nous présentons une interprétation de la systématisation des expériences du programme à la lumière de la perspective des postures de la connaissance de Balanzó, afin d'identifier les répertoires 
qui se produisent lorsqu'une œuvre artistique est encadrée dans des processus de travail institutionnels liés à des discours de politique publique.

\section{Mots clés}

Cirque ; théâtre ; clown ; construction de la paix ; droits de l'homme ; prévention de la consommation de substances psychoactives ; postures de la connaissance

Circo e teatro para a transformação social: repertórios de conhecimento e ação na construção de ambientes protetores e na promoção dos direitos das crianças e adolescentes

\section{Resumo}

Como o circo e o teatro podem contribuir para uma transformação social rumo à uma paz fundada na promoção dos direitos? Que lições recebemos das práticas de circo e teatro em contextos historicamente afetados pelo conflito armado? O que teatro, circo e clown precisam para se tornarem meios que facilitem a construção de ambientes de proteção para crianças e adolescentes nesses territórios? Este artigo aborda essas questões a partir da experiência do programa Circópópolis, transformando espacios y creando sueños, implementado em territórios afetados pelo conflito armado. Apresentamos uma interpretação da sistematização de experiências programáticas à luz da perspectiva das posturas de conhecimento de Balanzó, a fim de identificar repertórios que ocorrem quando uma obra artística é enquadrada em processos de trabalho institucionais vinculados a discursos de políticas públicas.

\section{Palavras-chave}

Circo; teatro; clown; construção da paz; direitos humanos; prevenção do consumo de substâncias psicoativas; posturas do conhecimento

\section{Circo i teatro trukachingapa socilapi: repertorio rigsilkuna rurangapa kauangapa uchullakunata} ariuambra, warmi wambra i musukunata

\section{Maillallachiska}

¿imasatak allika cirkua i tetroua aidangapa trukachingapa sumaglla kangapa chi promocionua Nokanchipa derechokuna? ¿ imata sakiska ka chi cirku i teatrokuna chi contextokuna kaskaska uagllichiska chi killaikuna sachukukuna? ¿ imatak ministiku teatro cirku i cliw kangapa chikuna aidangapa uñadangapa uñachingapa kauangapa uchullakunata i sipaskunata i musukunata kai territoriopi? kai uchulla kilkaskapi tapukumi imasatak chi programak circopolis, trukaska luar i uiñachirka muskuikuna,chi alpakunapi sakiskakuna sachukukuna.kauachinakunchimi sug interpretacionkuna sistematizacionpi chi programak punchaiachigsina churaska rigsiipi Balanzo kauangapa imatak luar, chi trabajo artisticok proceso trabajo institucional rimai politicokunaua publicupe.

\section{Rimangapa Ministidukuna}

Cirku teatro; clown; uiañachii; sumaglla karrii; Nukanchipa derechokuna; michai jiru ugsakuna mana chupanakuchu; posturas chi rigsiskakuna 


\section{Introducción}

El conflicto armado colombiano ha afectado al país por más de sesenta años, convirtiéndose en uno de los más antiguos y con más víctimas del mundo, extendiéndose a lo largo del territorio y en el tiempo (Centro Nacional de Memoria Histórica 2013, p. 111). De esta manera ha afectado proyectos de vida, proyectos comunitarios y el proyecto de un Estado democrático. Sin embargo, Colombia por décadas ha sido también el escenario de múltiples y diversas iniciativas comunitarias e institucionales de construcción de paz y memoria que, desde los territorios, han buscado aportar al proceso colectivo de tener un país sin guerra, que garantice los derechos humanos (Centro de Memoria Histórica 2013, p. 359).

Muñoz (2004, p. 14) habla de la paz como un proceso inacabado y agrupa en el concepto de paz imperfecta todas aquellas experiencias e instancias que han buscado regular los conflictos pacíficamente, en donde las personas han optado voluntariamente por la solidaridad, inclusive en contextos beligerantes. Desde el campo artístico también han sido múltiples las experiencias que buscan contribuir a este proceso, con diferentes alcances y propósitos. Una de ellas ha sido el programa Circópolis - transformando espacios y creando sueños, que desde 2017 ha utilizado al circo, el teatro y el clown como vehículos educativos, de movilización y sensibilización social. A través de metáforas, gestos, escenarios y prácticas, Circópolis busca fortalecer entornos protectores de niñas, niños y adolescentes, habilidades para la vida, competencias ciudadanas y proyectos de vida en municipios que han sido históricamente afectados por el conflicto armado en el país. Estas prácticas tienen como principal objetivo aportar a la promoción de los derechos de la niñez y la adolescencia, y prevenir su reclutamiento o utilización por parte de grupos armados, así como el consumo de sustancias psicoactivas. Este programa ha sido desarrollado en el marco de la Estrategia de Acciones Masivas de Alto Impacto Social (AMAS) del Instituto Colombiano de Bienestar Familiar (ICBF) en alianza con la Corporación Producciones La Ventana.

En este artículo analizaremos el programa, teniendo como base la sistematización de las experiencias de los años 2017 y 2018, a la luz del enfoque de Posturas del Conocimiento (Balanzó, 2018). Buscamos comprender los procesos de gestión del cambio a través del arte en estos contextos, de cara a la transición que atraviesan los territorios que históricamente han sufrido más la guerra. A partir de ahí, abrimos debates sobre los retos que implica para el ser creativo reconfigurar imaginarios, así como promover y fortalecer procesos de construcción de paz en los diferentes territorios. Nos interesa responder las siguientes preguntas: ¿Cómo se puede agenciar desde el circo-teatro social la transformación hacia una paz fundada en la promoción de los derechos? ¿Qué lecciones nos han dejado las prácticas circenses y teatrales en contextos que han sido históricamente afectados por el conflicto armado? ¿Qué exige al teatro, el circo y el clown ser un medio que facilite la construcción de entornos protectores para la niñez y la adolescencia en estos territorios?

El artículo se desarrolla de la siguiente manera: la primera sección describe la justificación y el marco institucional en los que se llevó a cabo el proyecto Circópolis, junto con sus estrategias de acción y contextos de ejecución. La segunda discute la metodología empleada para la sistematización del proceso y el enfoque conceptual de posturas de conocimiento a través del cual se realizó el análisis de la información. La tercera sección analiza el caso a la luz del marco conceptual, destacando los repertorios y los matices encontrados en los territorios y los aprendizajes centrales del programa. Por último, destacamos algunos elementos de interés en torno a la puesta de repertorios artísticos en torno al emprendimiento institucional de la paz.

\section{Circópolis y su contexto institucional}

Circópolis hace parte de las estrategias de Acciones Masivas de Alto Impacto Social, liderada por la Dirección de Niñez y Adolescencia del Instituto Colombiano de Bienestar Familiar, las cuales se enmarcan en la Política Pública de Infancia y Adolescencia. Este marco reconoce a las niñas, niños y adolescentes (NNA) como sujetos de derechos, actores en sus contextos, con capacidad de transformar sus entornos; como personas íntegras y capaces de participar en la vida pública y el desarrollo del país. En esta apuesta, los derechos de las niñas, niños y adolescentes son prevalentes, lo que obliga a toda la sociedad a velar por el bienestar y garantía de todos sus derechos siguiendo los lineamientos de la Convención Internacional de los Derechos del Niño, la Constitución Política de Colombia y el Código de la Infancia y la Adolescencia. En este contexto se busca proteger a los menores de edad de escenarios de riesgo como: 1 . violación, explotación sexual y económica, pornografía y acciones que atenten contra la libertad y la dignidad; así mismo, 2. guerras y conflictos armados y 3. reclutamiento y utilización por parte de grupos armados. Considerando esto, la 


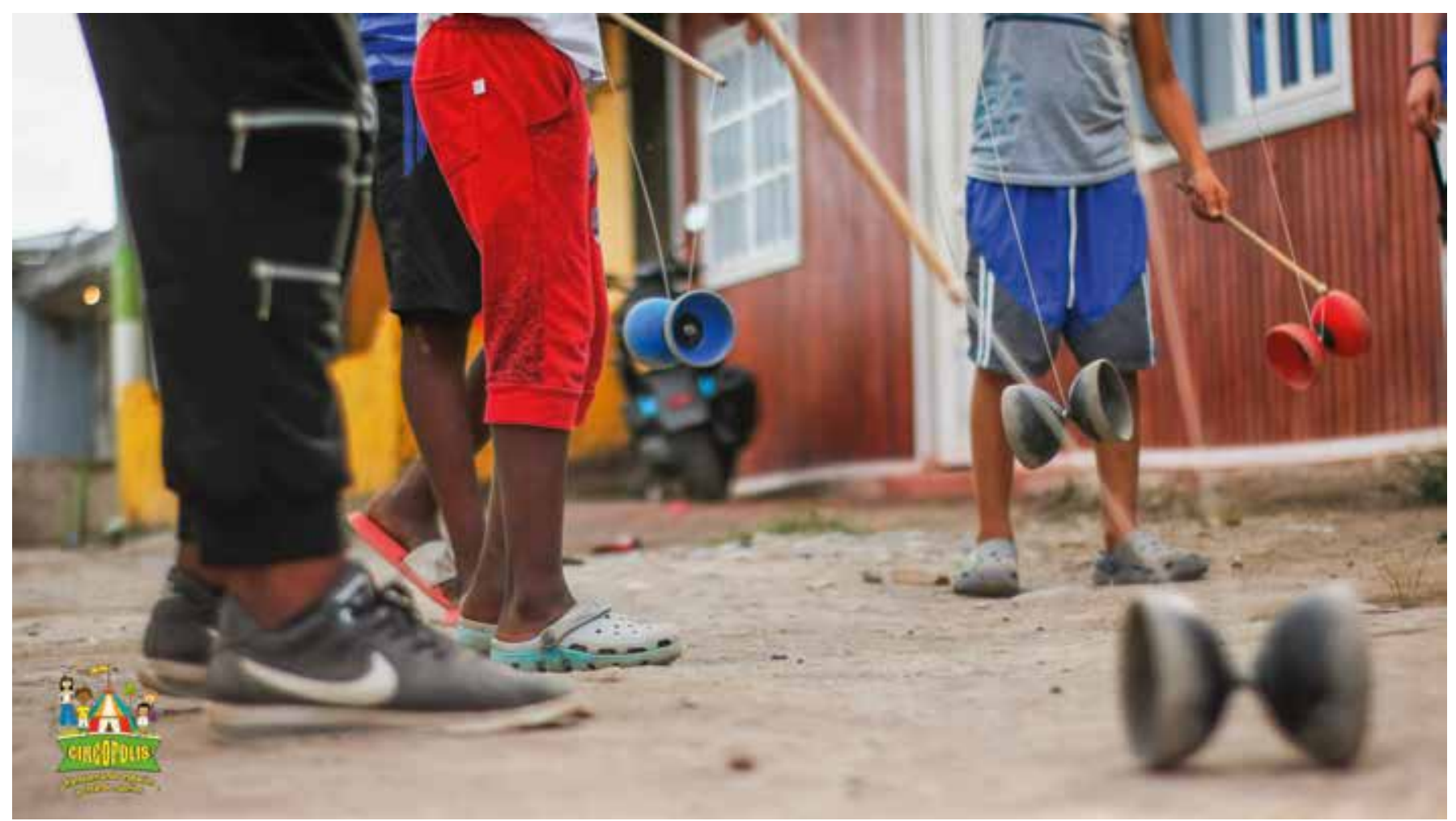

Imagen 1. Fotografía de la Corporación Producciones la Ventana (2019).

dirección de niñez y adolescencia, crea la Estrategia de Acciones Masivas de Alto Impacto Social (AMAS) con el objetivo de prevenir vulneraciones, promover los derechos de los NNA en contextos territoriales priorizados, a través de metodologías innovadoras y creativas, artísticas, culturales, deportivas y comunicacionales. Con estas estrategias se busca articular procesos de formación, movilización social y divulgación pedagógica como alternativas para el fortalecimiento de capacidades en los NNA y dinamización social de sus comunidades que promueva transformaciones necesarias dentro de los territorios desde la corresponsabilidad de todos los actores en el desarrollo integral de los NNA.

Circópolis utiliza el arte, específicamente el circo, el teatro y el clown, como escenario para facilitar el empoderamiento de NNA y en concordancia con la política pública y las prioridades de la Estrategia de Acciones Masivas de Alto Impacto Social (AMAS), el proyecto gira alrededor de los siguientes componentes: (I) Promoción de los derechos y empoderamiento. (II) Prevención de vulneraciones de derechos e identificación de riesgos. (III) Activación de rutas de atención para el restablecimiento de derechos vulnerados. (IV) Fortalecimiento de proyectos de vida. (V) Fortalecimiento de competencias ciudadanas, y (VI) La conformación y fortalecimiento de iniciativas y colectivos juveniles entre los participantes (Balanzó y Quiroga, 2018, p. 25).

La gestión de Circópolis incluye el desarrollo de un set de actividades alrededor de cada 'receta local'. Es decir, se busca convocar y movilizar actores y escenarios locales para fortalecer y propiciar el ejercicio de derechos y de NNA y los entornos protectores de los mismos. Por lo tanto, es un ejercicio que busca entrar en sintonía con las dinámicas locales y las iniciativas de gestión local existentes y potenciales. Todo esto se desarrolla a partir de varias premisas: 1 . un enfoque de derechos dirigido a la protección integral en un marco de corresponsabilidad social; 2. un enfoque poblacional en el que prevalezcan las características y dinámicas propias de cada territorio; 3. un enfoque de curso de vida, que retoma las transiciones, vivencia, roles y características particulares y comunitarias de las zonas en las que se desarrolla el proyecto; 4. un enfoque diferencial, que visibiliza y valora la diversidad de cada persona según sus condiciones particulares, contextos con los que interactúa y situaciones de vida, fundamentado en los principios de igualdad, diversidad, interculturalidad, integralidad, participación e inclusión; 5. un enfoque territorial, que reconoce el territorio como espacio contenedor de relaciones entre actores sociales, proyectos, objetivos de desarrollo y desarrollo de una identidad colectiva (Balanzó y Quiroga, 2018). 


\section{Contexto de ejecución de Circópolis}

En el año 2017, el programa Circópolis -transformando espacios y creando sueños - visita trece de los municipios en cuyas zonas rurales se ubican las Zonas Veredales Transitorias de Normalización, definidas en el marco del Acuerdo para la Terminación del Conflicto y la Construcción de una Paz Estable y Duradera (Alto Comisionado para la Paz, 2016). Así, Circópolis llega en el departamento de Caquetá a los municipios Cartagena del Chairá, Montañita, y San Vicente del Caguán; en el Cauca, hace presencia en los municipios de Buenos Aires, Caldono, y Miranda; en el Guaviare, se realizaron actividades en su capital, San José del Guaviare; en el Meta, se establece en los municipios de La Macarena, Mesetas, y Vistahermosa; en el departamento de Nariño va a los municipios Policarpa, Tumaco, y La Cruz; y, finalmente, en Putumayo el programa se desarrolla en el municipio de Puerto Asís. En cada uno de estos municipios, participaron por lo menos 193 niños, niñas y adolescentes del casco urbano.

En su versión 2018, Circópolis visita diez municipios que han operado como corredores territoriales estratégicos en la historia del conflicto armado y que comparten las particularidades de ser zonas de cultivos ilícitos, zonas de tránsito para la industria del narcotráfico y tener la presencia activa de grupos armados ilegales, disidencias de los mismos o grupos delincuenciales organizados. El programa llega durante seis meses al departamento de Córdoba, en los municipios de Montelíbano, Puerto Libertador y San José de Uré; en Santander se hace presencia en Barrancabermeja; en el Sur del departamento de Bolívar se llegó a Cantagallo, San Pablo, Santa Rosa del Sur y, finalmente, en el departamento de Guaviare se llegó a los municipios de El Retorno y Calamar y se retomó el trabajo realizado en el años 2017 en la capital, San José del Guaviare, incluyendo a dos de sus corregimientos: Puerto Nuevo y Cachicamo, siendo zonas priorizadas cercanas a la cabecera municipal. En cada uno de estos municipios participaron por lo menos 230 niños, niñas y adolescentes, tanto del casco urbano y como de una de sus veredas, la cual focalizada por el proyecto en articulación con los actores locales.

En suma, el proyecto ha involucrado a más de cinco mil niños, niñas y adolescentes, y ha articulado a distintos actores, tales como educadores, autoridades locales y familias, con quienes se ha propendido por la construcción y fortalecimiento de entornos protectores para niñas, niños y adolescentes. De manera general, los municipios a los que se ha llegado comparten las siguientes características, aunque se generan dinámicas diferenciadas al respecto (Balanzó y Quiroga, 2018):

- Antecedentes (o vigencia) de la presencia de grupos armados al margen de la ley y la lucha entre ellos por obtener el control territorial.

- Expansión de economías ilegales: hay zonas de cultivos de uso ilícito o se desarrollan actividades extractivas de manera informal. También hay corredores fluviales o terrestres de narcotráfico, en algunos casos hay también laboratorios de procesamiento de coca. El control de estas economías es fuente de conflicto, afectando el tejido social.

- Multiculturalidad: Varios de estos territorios son habitados por pueblos indígenas y/o han sido poblados por personas provenientes de varias zonas del país en procesos de colonización tardía o por causa del desplazamiento forzado que se ha dado a nivel nacional.

\section{Estrategias de acción de Circópolis. ¿Cómo crear entornos protectores de los derechos de los NNA a través del teatro y el circo?}

A estos municipios llega Circópolis a realizar actividades en cuatro componentes: formación, movilización social, divulgación pedagógica y sistematización.

El componente de formación tiene como objetivo desarrollar en los NNA una serie de habilidades para la vida y competencias ciudadanas que les permita pensarse en sus contextos como sujetos de derechos, a través de un ciclo de talleres de circo y teatro-clown. Se realiza un ejercicio de prevención positiva que parte de la conciencia que los derechos nacen del reconocimiento del otro y se interiorizan desde el afecto: el afecto por mí mismo y por los otros. En este ejercicio el cuerpo es el espacio primario de las emociones y los aprendizajes. En su versión 2018 el 91\% de los niños, niñas y adolescentes participantes del programa asistió a dieciocho talleres de circo y dieciocho talleres de teatro-clown, permaneciendo en el proceso desde la primera hasta la última clase.

El componente de movilización social busca que los niños, niñas y adolescentes del proyecto, como sujetos de derechos y sujetos políticos, compartan sus creaciones y entablen diálogos sobre las percepciones y preocupaciones que les generan sus entornos familiares, sociales, educativos, artísticos, políticos y ambientales con los adultos que intervienen de manera significativa en sus procesos de crianza, cuidado y protección. Estos espacios buscan construir alternativas colectivas de 
transformación de las realidades locales y sobre todo crear compromisos en torno al afecto, el cuidado, el respeto y los derechos de la niñez y la adolescencia. Las actividades de este componente son: caravanas de lanzamiento, teatros foro, encuentros con docentes, encuentros con la comunidad, encuentros con las familias, funciones de acompañamiento y funciones de cierre; ocasionalmente también se crean zonas libres de consumo de alcohol y cigarrillo durante el desarrollo de las ferias y fiestas de los municipios. A continuación, describiremos brevemente cada una de estas actividades:

- Caravanas de lanzamiento: En cada municipio, Circópolis realizó una caravana artística con el fin de presentar el programa a la comunidad y realizar una jornada de inscripción para los NNA.

- Teatros foros: En cada territorio se realizaron tres teatros foros, dos en la cabecera municipal y uno en la vereda. En cada uno de estos teatros foros, los NNA construyeron escenas en las que abordaban sus percepciones sobre la vulneración de derechos y el consumo de sustancias psicoactivas en sus contextos. A partir de estas escenas, se fomenta el diálogo entre padres, madres, cuidadores, docentes, niños, niñas y adolescentes con el fin de acordar compromisos concretos hacia la promoción de entornos protectores

- Funciones de acompañamiento: Se realizó una función de artistas profesionales en cada uno de los municipios y veredas como apoyo metodológico del proceso artístico y motivacional al componente de formación.

- Encuentros con la comunidad: Se realizó un encuentro artístico con la comunidad en cada uno de los municipios. Estos encuentros son tomas artísticas que generan el encuentro de los participantes del programa con diversos actores de sus comunidades, en donde aquellos pueden compartir lo que han aprendido en el proceso de formación del programa.

- Encuentro con docentes: Se realizaron dos encuentros con docentes en cada uno de los municipios; en ellos se comparte la metodología artística con enfoque de derechos.

- Encuentro para las familias: Se realizó un encuentro con familias para cada uno de los municipios y veredas, que consistieron en jornadas lúdicas que buscaron propiciar un encuentro desde el afecto entre los padres, madres y cuidadores y niñas, niños y adolescentes participantes, con el fin de fortalecer entornos protectores.

- Funciones de cierre: Los niños, niñas y adolescentes participantes del proyecto de las cabeceras municipales y veredas realizaron una presentación artística para el cierre del componente de formación. Estas funciones se convierten también en el espacio donde el proyecto pasa a ser gestionado directamente por las comunidades, por lo que se les entregan los materiales de prácticas circenses.

- Por último, en el marco de algunas ferias y fiestas municipales, Circópolis instala un espacio llamado "Zonas libres de consumo de alcohol y cigarrillo", un espacio de circo y teatro orientado a los niños, niñas y adolescentes y a sus cuidadores, con el fin de posicionar la discusión sobre la creación de espacios culturales para los menores de edad.

El componente de divulgación pedagógica busca comunicar las apuestas, los procesos pedagógicos, los aprendizajes y logros del programa inicialmente entre los NNA participantes y sus comunidades, extendiéndose a los demás actores interesados. Para esto se diseñó un plan comunicativo que incluye la difusión por redes sociales, la creación de una canción y la producción de una serie de videos edu-comunicativos.

Finalmente, el componente de sistematización pone a disposición del proyecto y de las entidades interesadas en el mismo, instrumentos para su seguimiento y evaluación que sirvan como base en la toma de decisiones y provea insumos para las memorias del proceso. Sobre este componente profundizaremos a continuación.

\section{Aspectos metodológicos y conceptuales de la sistematización de Circópolis}

Esta investigación se basa en la sistematización de los procesos generados en la ejecución de Circópolis en sus versiones 2017 y 2018 . Como tal, cada sistematización buscó realizar una lectura interpretativa de realidades y dinámicas locales en términos de los aprendizajes, las transformaciones logradas, las tensiones y los conflictos con los que se enfrentó el proyecto, y los retos hacia el futuro. Todo este análisis se hace en relación con los objetivos del programa, de las Estrategias AMAS y de los componentes de política pública en los que se enmarca. Para una lectura reflexiva de la experiencia se hizo uso de herramientas de seguimiento y monitoreo que permitieran la captura de datos cualitativos. Estos incluyen:

- La relatoría de eventos y puestas en escena del componente de movilización social. 


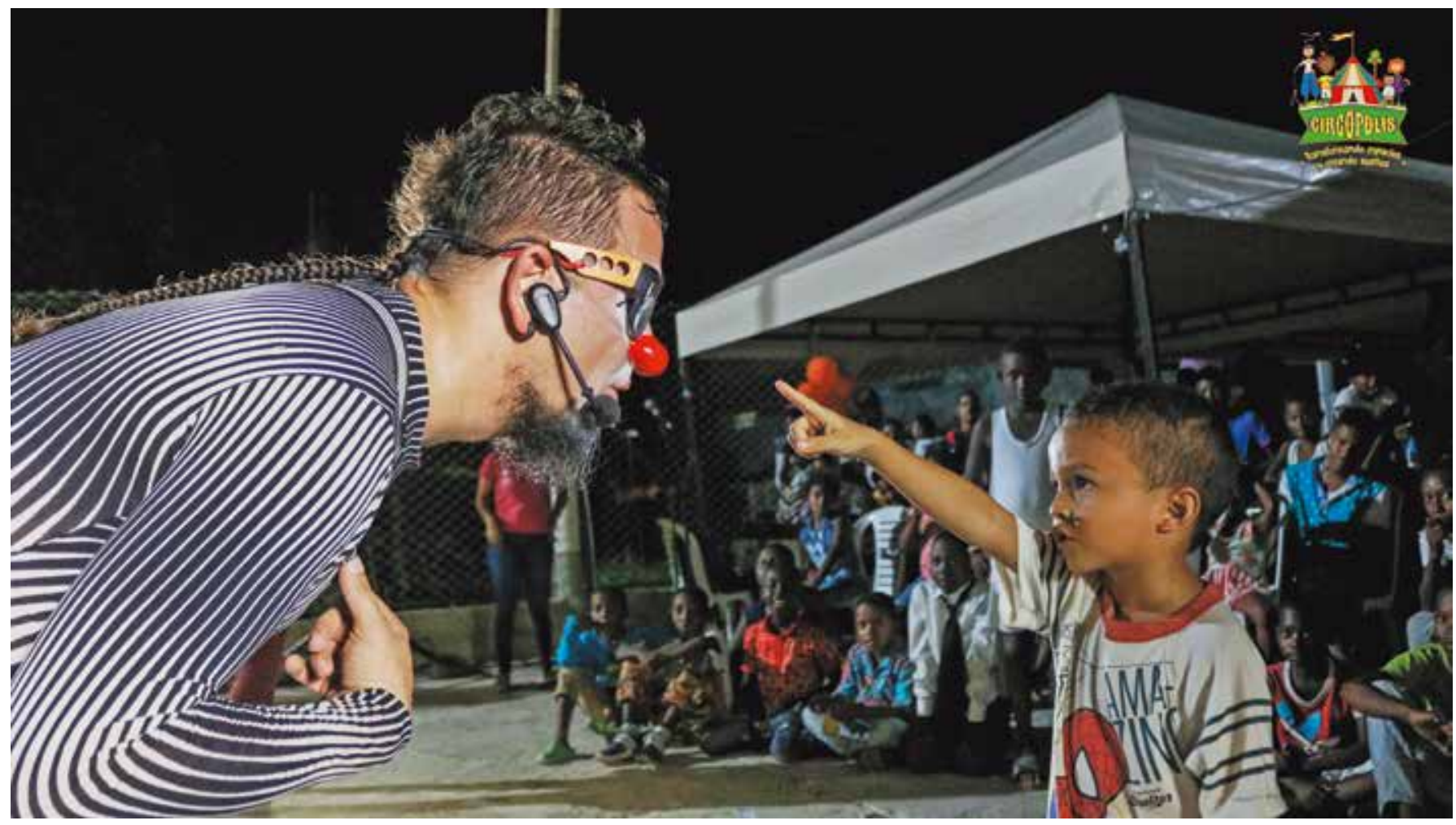

Imagen 2. Fotografía de Corporación Producciones la Ventana (2019).

- Un primer diagnóstico del contexto, realizado a través de una cartografía social que ofrece una lectura del entorno en términos de realización de derechos y de factores de riesgo y protección en relación al consumo de sustancias psicoactivas y el reclutamiento y utilización de NNA.

- Un segundo diagnóstico psicosocial que, de acuerdo con la experiencia que el equipo ha tenido en el territorio, profundiza sobre el estado de la protección de derechos de la infancia y la adolescencia en varios ámbitos: hogar, salud, educativo, público o comunitario y virtual.

- Una última evaluación que se realiza por medio de un grupo focal con los facilitadores de teatro y circo, de acuerdo a las actividades pedagógicas implementadas en referencia con la Guía Metodológica (2017).

- La realización de bitácoras semanales para cada territorio en las que los equipos hacen relatorías sobre los procesos de gestión del proyecto y las dinámicas sociales locales.

\section{Discusión: agencia del cambio de Circópolis}

En esta sección realizaremos una lectura de Circópolis a la luz del enfoque conceptual de posturas de conocimiento en aras de identificar repertorios que tienen lugar cuando el trabajo artístico se enmarca en procesos de trabajo institucional ligados a discursos de política pública.

\section{Marco conceptual: el enfoque 'posturas de conocimiento'1}

El enfoque de 'posturas de conocimiento' (knowledge stances perspective) (Balanzó, 2016) nos brinda herramientas para analizar la agencia del cambio², aportando detalles sobre sus dimensiones cognitivas, prácticas e institucionales. A través de este enfoque interpretamos la información recolectada durante la sistematización de los procesos de Circópolis en sus versiones 2017 y 2018.

¿Qué implica generar cambio? Los agentes de cambio son "líderes, grupos, coaliciones u otros que pueden

1 El presente texto está basado en una traducción de la sección
de marco teórico de Ramos-Mejía y Balanzó (2018). What It Takes
to Lead Sustainability Transitions from the Bottom-Up: Strategic
Interactions of Grassroots Ecopreneurs. Sustainability, 10(7), 2294;
https://doi.org/10.3390/su10072294. Agradecemos a Juan Pablo
Centeno la traducción.
$2 \quad$ La agencia del cambio "describe una variedad de repertorios
en búsqueda de crear, estabilizar y expandir campos de práctica
específicos" (Balanzó, 2016, p. 38).


iniciar y conducir transformaciones hacia el logro de un objetivo de desarrollo" (Roberts y Ling, 2012, p. 11). Esta definición resalta el potencial de los actores para promover el cambio institucional (DiMaggio, 1988), esto es, para afectar prácticas sociales y reglas del juego. Balanzó (2016) identificó cinco estrategias distintas que se despliegan para actuar como agentes de cambio: 1) actuar con consistencia interna, 2) extender el campo de práctica, 3) eludir cuellos de botella y reescalar, 4) intermediar ciclos de conocimiento y 5) jugar un rol en la esfera pública.

Primero, los actores actúan con consistencia interna cuando su comportamiento intencionalmente refleja valores y motivos específicos, demostrando y expresando una identidad específica. En el caso de Circópolis hemos expuesto brevemente sus objetivos, enfoques y líneas de acción en los apartados anteriores. Seguiremos analizando el caso en relación con las demás estrategias en el próximo apartado.

La segunda estrategia consiste en crear alianzas para colaborar y alimentar un campo de práctica con el fin de extenderlo o profundizarlo. La tercera, eludir cuellos de botella y re-escalar, usualmente busca posicionar temas en otras escalas, llamando la atención de un espectro más amplio de actores. La cuarta estrategia, intermediar ciclos de conocimiento, se refiere a la adquisición de nuevo conocimiento y su traducción en conceptos comprensibles y operables en la práctica. Finalmente, los actores juegan un rol en la esfera pública cuando actúan más allá de su propio campo, involucrándose en la deliberación pública para acceder a espacios de debate más amplios y ganar apoyo o impactar el diseño de políticas.

Balanzó (2016) desarrolla un marco conceptual comprensivo que busca señalar repertorios posibles de agencia que pueden ser identificados en el intento de generar cambio. El autor describe este tipo de agencia como actos de posicionamiento (Downey, 1992), en el que las estrategias surgen como formas prácticas de adaptación para hacer frente a las situaciones (Chia y Holt, 2006). De tal suerte que las estrategias responden a las posiciones relativas de los agentes en sus contextos. Las posturas de conocimiento constituyen una heurística para abordar los repertorios de un agente: en suma, los gestos que despliega a fin de lograr el cambio. A continuación, la explicación de cada postura de conocimiento:

- Exploración de frontera: las estrategias de exploración de frontera se refieren a estrategias de adaptación en las que actores colaboran con pares u otros aliados.

1. Gestión de la práctica: describe aquellos arreglos y actividades que se ponen en marcha para promover, hacer posible, sostener en el tiempo y moldear las racionalidades, reglas, rutinas y valores de un campo de práctica.

2. Demarcación de frontera: describe la situación y acciones de un actor en torno a aquellos límites que dan marco de restricción o posibilidades para la agencia. Estos límites pueden surgir de relaciones de poder frente a otros actores (situaciones de frontera), tanto como a reglas del juego existentes (condiciones de frontera). La demarcación de frontera se refiere a las estrategias de reacción que permiten ampliar, zanjar, imponer, transformar o derribar esos límites.

3. Intermediación de conocimiento: se refiere a las formas de trabajo del conocimiento (esto es, almacenamiento, manipulación y oferta de conocimiento) orientadas a nutrir un campo de práctica.

4. Oferta de conocimiento: se refiere a la consecución y ubicación de aquellos conocimientos que nutren las distintas posturas. Los contenidos de oferta de conocimiento incluyen todos los tipos de conocimiento: por ejemplo, formas de conocimiento local, contextual, objetual, técnico, experto y/o científico y pueden, por tanto, nutrir las distintas estrategias que tienen lugar allí.

5. Exploración de conocimiento son los procesos

6. de develar y producir conocimiento.

Balanzó (2016) discute algunas dimensiones que son comunes a las posturas del conocimiento. Estas dimensiones están relacionadas con cuatro dominios dados, transversales a la agencia. El primer dominio está relacionado con un dominio existencial, que marca los pilares centrales de los agentes como actores. El segundo dominio es normativo, referido a la agencia con (y sobre) reglas, normas y regulaciones que hay dentro y fuera de las organizaciones. El tercer dominio se refiere a la interacción, que en un sentido amplio trata sobre las acciones en las que los actores se relacionan con otros actores o campos, buscando, compartiendo, creando, refutando o protegiendo los medios para ocuparse de una práctica. El cuarto dominio hace alusión a la práctica, incluyendo los arreglos de actividad colaborativa para fortalecer un campo de práctica. El último dominio es el cognitivo, este se refiere a la agencia que produce y reproduce conocimiento para sí mismo y para un campo de práctica. 


\section{Circópolis a la luz de este enfoque conceptual}

Para el caso de Circópolis el fortalecimiento de entornos protectores de los derechos de niñas, niños y adolescentes configura el campo de práctica al cual aporta el proyecto. Su ámbito de trabajo institucional tiene que ver con los actores y referentes normativos extralocales y locales con los que tiene relación en función de profundizar el alcance del ejercicio. Este ámbito se conoce informalmente en Colombia como el ámbito de la gestión. El ámbito de intermediación de saberes aquí tiene que ver con los repertorios puestos en marcha por Circópolis para configurar espacios de realización, utilizando como medio las artes y metodologías escénicas.

Puede que el mayor aprendizaje de Circópolis es que no hay fórmula única para abordar los diferentes contextos con los que se interactúa. Si bien se adoptó un enfoque territorial, las realidades locales exceden toda presunción inicial. Esto implica conservar la perspectiva en cuanto a la evaluación de los impactos generados y los modos de acción. Estos ritmos y dinámicas locales afectan desde aspectos logísticos como el manejo de los espacios y los horarios, hasta aspectos como los valores y las creencias con las que se entra a dialogar.

\section{Comenzaremos el análisis con la Exploración de}

Frontera. Cabe recordar que la Exploración de Frontera describe aquellas estrategias de adaptación en las que actores colaboran con pares $u$ otros aliados. Para el caso, el convenio con el programa AMAS supone un primer espacio de exploración de frontera. En torno a aquel se configuran distintos momentos de exploración de la configuración de recetas locales, con distintos grados de formalidad. Por ejemplo, considerando varios de estos municipios gran parte de la oferta institucional para la infancia y adolescencia llega desde una instancia central sin proyecciones de continuidad y sin mecanismos de anclaje con experiencias previas. Los equipos en territorio entraron a participar de las diferentes reuniones concertadas en cada municipio alrededor del tema de niñez y adolescencia, como la MIAF (Mesa de Infancia, Adolescencia y Familia), COMPOS (Consejo Municipal de Política Social) y PDTS (Planes de Desarrollo Territorial), realizando avances significativos en términos de gestión interinstitucional. Esto se ha materializado en acciones concretas para la garantía, la protección y el restablecimiento de los derechos de las niñas, niños y adolescentes. En San José de Uré se logró incluir, dentro de los PDTS que parte de la inversión municipal estuviera orientada a la promoción de jornadas extraescolares, la enseñanza del arte, la construcción y dotación de espacios para la transmisión de la cultura, y la creación de espacios permanentes dedicados a la protección y prevención en pro de la reconciliación. Además, en San José de Uré y San José del Guaviare se abrió un diálogo entre Circópolis y los saberes locales de las comunidades indígenas y afrodescendientes: las niñas, los niños y los adolescentes que pertenecen a Circópolis se articularon a otros grupos como el de danzas y tambores para realizar creaciones colectivas.

El proyecto incluye también ejercicios de formalización alrededor de la apropiación local de los activos de Circópolis, con la firma de acuerdos con los cuales otros actores que desarrollan roles de gestión a la par con Circópolis. Por ejemplo, en Puerto Libertador y El Retorno se realizó un ejercicio de articulación con las emisoras municipales en donde a través del radioteatro niñas, niños y adolescentes hacen promoción de sus derechos. Además, en los municipios de Cantagallo, San Pablo, Puerto Libertador y San José de Uré grupos de teatro conformados por jóvenes de manera autónoma, que venían realizando sus prácticas desde lo empírico, empezaron a articularse con los equipos en territorio de Circópolis y fortaleciendo su formación y su capacidad de gestión. Cuando el equipo de Circópolis terminó sus labores, la anterior iniciativa facilitó la continuidad del proceso en estos territorios.

Otras situaciones de exploración de frontera tienen que ver con la disposición de los espacios físicos en los que opera Circópolis. Interesa destacar aquellas que tienen que ver con participación en arreglos locales participativos: en el municipio de Santa Rosa del Sur se recuperó un coliseo de alto rendimiento, que, aunque contaba con salones amplios, un auditorio y una cancha para la práctica de baloncesto y fútbol-sala, no contaba con servicio de electricidad, agua, mantenimiento y seguridad (Balanzó y Quiroga, 2018, p. 56). La llegada del programa generó la atención en las entidades municipales, logrando que estas deficiencias se suplieran, beneficiando no solo a los participantes de Circópolis sino a la comunidad en general.

Finalmente, otra exploración de frontera tiene que ver con la familia. En este punto es importante anotar que los modelos y situaciones familiares encontrados en territorio eran diversos. Por ejemplo, en la vereda de San Pablo, el Encuentro con la Familia se realizó con los compañeros y compañeras del internado de los participantes de Circópolis. En todos los municipios se registró una baja asistencia de las familias en las 
actividades de movilización, al indagar por las razones, las niñas, niños y adolescentes manifiestan que se debe al trabajo, al desinterés en las actividades o a que simplemente ellos no tenían conocimiento porque no existen canales de comunicación efectivos dentro del hogar. Además, para varias de estas familias el castigo físico sigue siendo cotidiano, por esta razón tanto los NNA como sus cuidadores se sorprenden con esquemas de relacionamiento que parten desde el afecto. Además, fue necesario intermediar el hecho de que algunos padres empezaran a interponer como castigo a sus hijos e hijas el no ir a los talleres de Circópolis.

La segunda postura de conocimiento es la Gestión de la Práctica. La gestión de la práctica describe aquellos arreglos y actividades que se ponen en marcha para promover, hacer posible, sostener en el tiempo y moldear las racionalidades, reglas, rutinas y valores de un campo de. Aquí es necesario subrayar que el campo de práctica en este caso es aquel de la realización de derechos mediante el circo teatro y el clown, y no aquel de la enseñanza artística.

La gestión de la práctica es visible en Circópolis en todas aquellas gestiones orientadas a profundizar la realización de derechos en los ámbitos inmediatos y mediatos del proyecto. Entre ellos, los mecanismos de relacionamiento previstos con docentes y comunidades, para efectos de crear espacios de diálogo sobre las preocupaciones de los NNA, los retos locales, el alcance de metodologías empoderadoras y la vinculación activa de actores a los procesos. Por ejemplo, en el I Teatro Foro de San José de Uré los niños, niñas y adolescentes crearon escenas sobre la violencia intrafamiliar, el acoso escolar, violencia contra la mujer y falta de espacios para la recreación. En el proceso de creación también salieron otros temas como el consumo de sustancias psicoactivas, la discriminación, el racismo y la participación en grupos armados (Balanzó y Quiroga, 2018, p. 36). En tercer lugar está la demarcación de frontera, que describe la situación y acciones de un actor en torno a aquellos límites que dan marco de restricción o posibilidades para la agencia. Estos límites pueden surgir de relaciones de poder frente a otros actores (situaciones de frontera), tanto como a reglas del juego existentes (condiciones de frontera). La operación de Circópolis no abunda en repertorios de demarcación de frontera, aunque hay de ello algo implícito en la promoción de derechos. Los aspectos visibles aparecen en virtud de su condición de rostro visible del ICBF: aquellos que tienen que ver con la activación de rutas de atención. Se presentan múltiples limitaciones en la activación de estas rutas, por ejemplo, por la ausencia

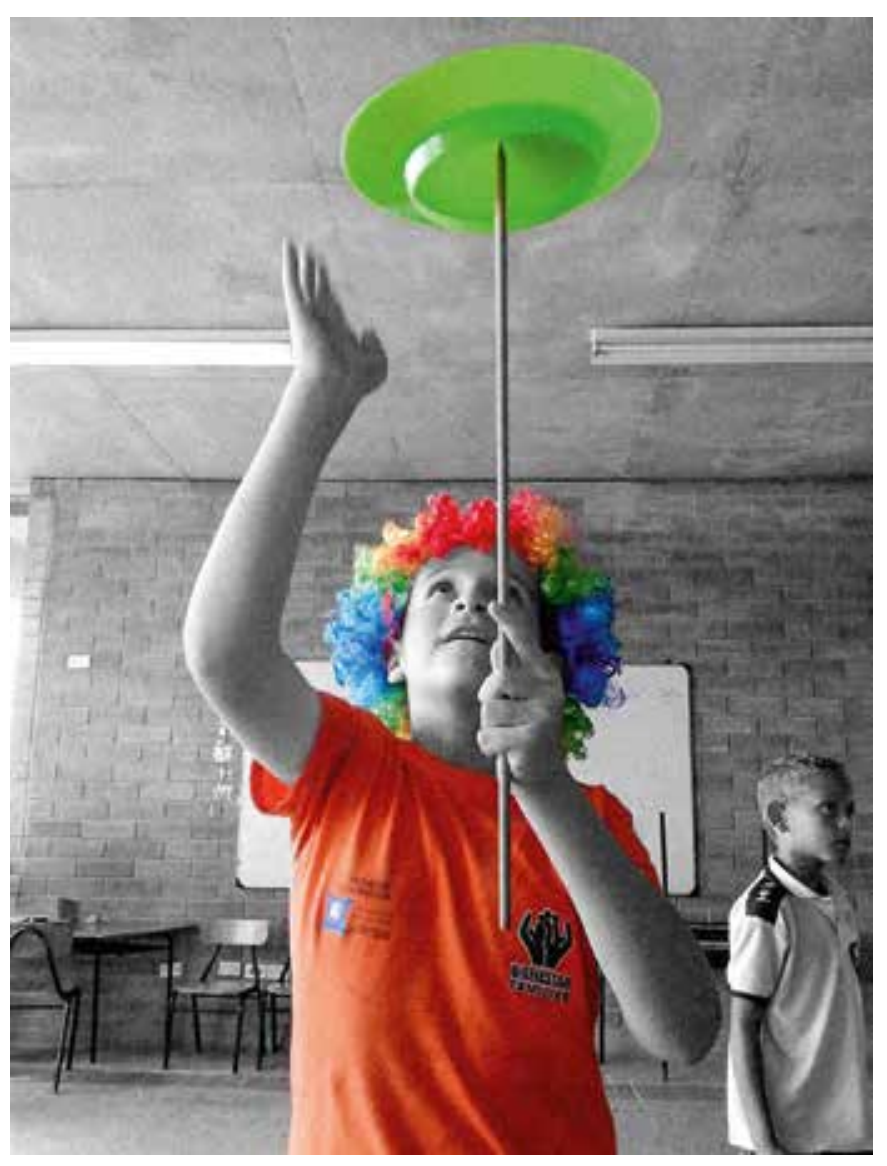

Imagen 3. Fotografía de Corporación Producciones la Ventana (2019).

de especialistas en los municipios que den respuesta oportuna a cada uno de los casos. Además, en algunos territorios representó un riesgo para el equipo activar estas rutas, recibiendo amenazas consecuencia de mal manejo de la información que hicieron los funcionarios públicos que recibieron la denuncia.

No obstante, hay casos como el del municipio de Puerto Libertador, donde se logró que se destinará dentro del COMPOS (Consejo Municipal de Política Social) un rubro de 50 millones para el fortalecimiento del Equipo de la Comisaría de Familia en veredas y corregimientos donde no hace presencia; con ello se pretende fortalecer la atención efectiva en situaciones de vulneración de derechos. En este municipio también se adoptó dentro de la mesa de educación de los Planes de Desarrollo Territorial (PDTS) la propuesta de construir un plan municipal que integre a niñas, niños y adolescentes en condición de discapacidad, y abrir áreas de psico-orientación en las instituciones educativas. (Balanzó y Quiroga, 2018, p. 64)

Respecto a la intermediación de conocimiento, referido a las formas de trabajo del conocimiento orientadas a nutrir un campo de práctica. El principal activo de 


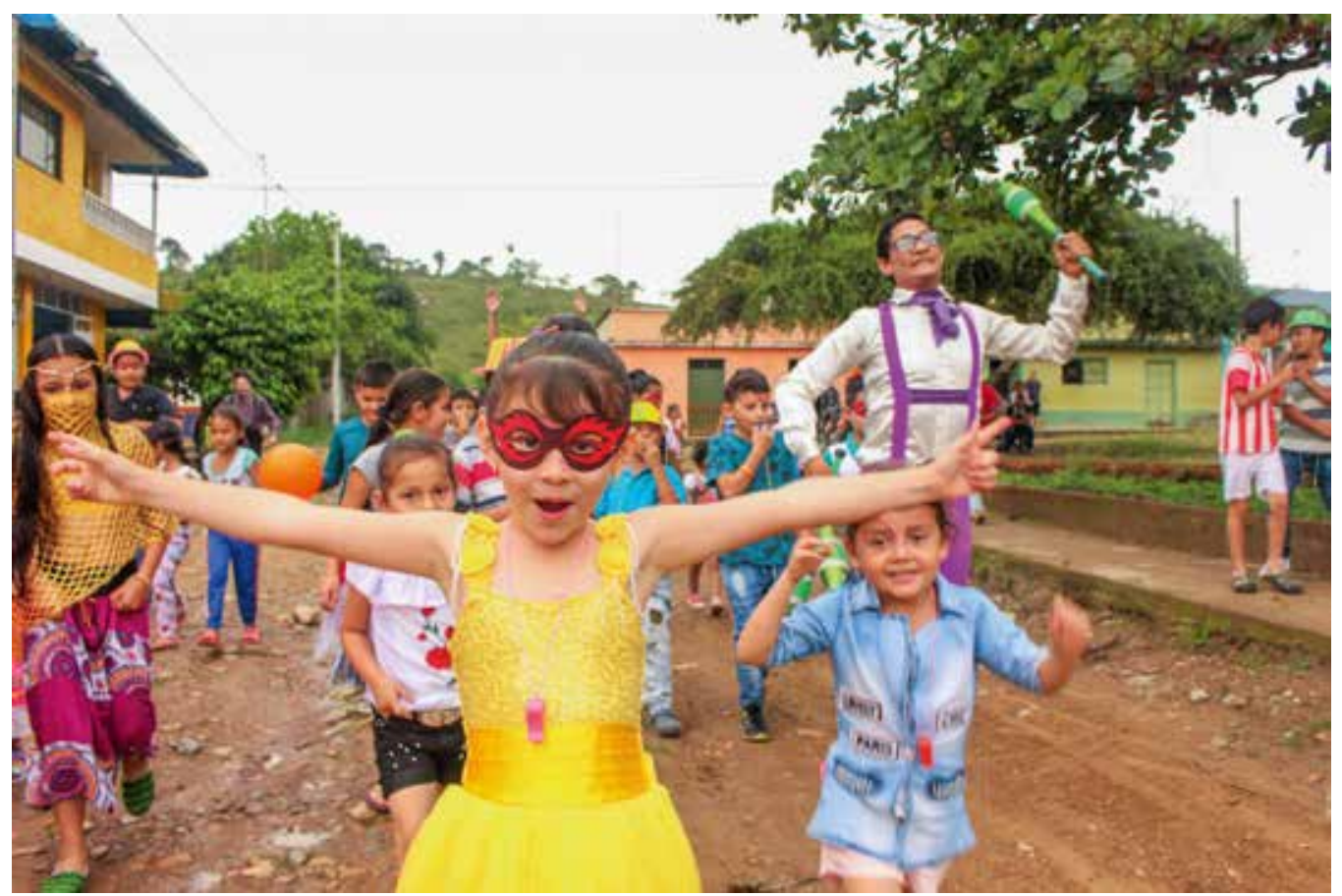

Imagen 4. Fotografía de Corporación Producciones la Ventana (2019).

Circópolis tiene que ver precisamente con su capacidad de intermediación en la frontera entre la promoción y realización de derechos de los NNA y la enseñanza, puesta y producción en torno a las artes escénicas. Quizás el objeto más concreto de este repertorio es la Guía Pedagógica creada en 2017. No obstante, ha sido claro en los ejercicios de evaluación 2017 y 2018 que la guía establece tan solo una referencia frente a la cual los facilitadores desarrollan didáctica y procesos acordes con los entornos locales, para actividades de formación tanto como de movilización. Interesa notar que la evolución del proyecto ha tenido que ver precisamente con ampliar los repertorios de intermediación hacia actores relevantes en el entorno de los NNA: comienza con un interés inicial en docentes, y se extiende después hacia familias (2017) y gestores culturales (2018). El foco de ese repertorio tiene que ver con poner en juego situaciones de realización de derechos a partir del juego metodológico tanto como de las voces de los NNA.

En este punto destacamos los aprendizajes del componente más grande del programa: la formación. Los logros artísticos fueron evidentes: niños, niñas y adolescentes haciendo un despliegue circense y teatral en sus territorios. Destacamos un segundo nivel de aprendizaje al que se llegó a través del diálogo y la articulación entre las técnicas circenses y teatrales con aspectos psicosociales y que generó cambios sustanciales a nivel cognitivo, emocional y relacional: mayor autoestima, autonomía en la toma de decisiones, expresión asertiva de emociones, pensamiento crítico, cooperación y trabajo en equipo, empatía, resolución de conflictos, liderazgo, optar por estilos de vida saludable, entre otros.

El segundo repertorio de intermediación es la oferta de conocimiento. En el caso de Circópolis la principal función de oferta tiene que ver con la puesta de expertos en escenarios de facilitación. Se suma a ello el desarrollo de una premisa metodológica que pasa por sintonizar contenidos y métodos escénicos en pos de la promoción de derechos. En la práctica esto pasa por el uso de metáforas, la captura y trabajo con experiencias locales, la puesta en valor a través de la escena de la voz de los NNA. En interacción con otros actores involucrados la fuente de conocimiento tiene que ver con inspirar la realización de derechos en los espacios cotidianos de vivencia y educación: ese modo de trabajo de la práctica que consiste en ser ejemplo del cambio deseado.

El último repertorio de intermediación es aquel de la exploración de conocimiento: el proceso de develar y producir conocimiento. En el caso de Circópolis el principal espacio de exploración tiene que ver con el desarrollo metodológico in situ, en el escenario de enseñanza y producción colaborativa con NNA. En territorio, la vivencia escénica adquiere otros matices de intercambio social, es el ejercicio de desarrollo exploratorio de temas, puestas y roles en común. 


\section{Conclusión: El reto estructural de construir paz con enfoque de derechos a través del arte}

¿Cómo se puede agenciar desde el circo-teatro social la transformación hacia una paz fundada en la promoción de los derechos? ¿Qué lecciones nos han dejado las prácticas circenses y teatrales en contextos que han sido históricamente afectados por el conflicto armado? ¿Qué se le exige al teatro, el circo y el clown para ser medios que faciliten la construcción de entornos protectores para la niñez y la adolescencia en estos territorios? En este artículo abordamos esta serie de preguntas desde la experiencia del programa Circópolis, desarrollado como parte de las Estrategias AMAS del ICBF en alianza con la Corporación Producciones La Ventana. Esta experiencia nos ha dejado una serie de aprendizajes y, sobre todo, nos ha delineado los retos estructurales de la construcción de paz con enfoque de derechos a través del arte.

Como lo expusimos anteriormente, las artes son capaces de cambiar escenarios e imaginarios, los NNA participantes del programa se han apropiado de espacios deteriorados para crear nuevas historias. En territorios marcados por el conflicto, las artes siguen aportando a la creación y descubrimiento de otros posibles caminos, cuerpos y voces; en donde antes hubo enfrentamientos hoy se construye en colectivo. De Circópolis queda la convicción de saber que es posible gestionar y transformar realidades en la búsqueda de fortalecer entornos protectores de los derechos de la infancia y la adolescencia. Nos queda la certeza de no tener una fórmula que funcione en cada lugar al que llega el programa, pero sí de tener herramientas que permitan pensar en estrategias a la hora de enfrentar nuevos retos. Asimismo, queda el desafío de haber encontrado una serie de limitaciones que pueden enriquecer las nuevas puestas en marcha del programa ¿Cómo hacer una activación de rutas para la protección de derechos de los NNA más efectiva? ¿Cómo movilizar en mayor medida a profesores, familias y cuidadores, y cómo hacer que estos asuman compromisos concretos en el fortalecimiento de entornos protectores de los derechos de los NNA? ¿Cómo hacer una sistematización que recoja efectivamente los aprendizajes pedagógicos en el área de teatro y circo, en diálogo con cada uno de los territorios?

\section{Referencias}

Alto Comisionado para la Paz (2016). Acuerdo final para la terminación del conflicto y la construcción de una paz estable y duradera. Bogotá: Colombia.

Araque Osorio, C. (2015). El personaje de la libertad cómica. Calle 14 Revista De investigación En El Campo Del Arte, 10(16), 43-54. https://doi. org/10.14483/10.14483/udistrital.jour.c14.2015.2.a04

Balanzó, A. (2016). Unfolding Capacity: Strategies of Farmers' Organizations as Change Agents. The Netherlands: University of Twente.

Balanzó Guzmán, A. y Quiroga, D. (2018). Sistematización de la experiencia: Circópolis, transformando espacios y creando sueños. Bogotá: ICBF.

Centro Nacional de Memoria Histórica, (2013). iBASTA

YA! Colombia: Memorias de Guerra y Dignidad. Colombia: Memorias de guerra y dignidad. Bogotá: Imprenta Nacional.

Chia, R., y Holt, R. (2006). "Strategy as Practical Coping: A Heideggerian Perspective". 27, 635-655. https://doi. org/10.1177/0170840606064102

DiMaggio, P. J. (1988). "Interest and Agency in Institutional Theory", en L. G. Zucker (Ed.), Institutional Patterns and Organizations: Culture and Environment, pp. 3-21. Ballinger: Cambridge, MA.

Downey, Gary Lee (1992). "Human Agency in CAD/CAM Technology", en Anthropology Today, 8, 2-6. https://doi. org/10.2307/2783567

Muñoz, F (2004) "Paz imperfecta", en Enciclopedia de paz y conflictos 2 (2004). Granada: Universidad de Granada

Roberts, D., y Ling, C. M. (2012). “Guide to Evaluating Capacity Development Results: A Collection of Guidance Notes to Help Development Practitioners and Evaluators assess Capacity Development Efforts". Washington, D.C.: Banco Mundial. Disponible en: http://documents. worldbank.org/curated/en/352241468163166112/ pdf/762800WPOBox370evelopment0Results00.pdf 